\title{
Effect of Riboflavin/UVA Collagen Cross-linking on Central Cornea, Limbus and Intraocular Pressure. Experimental Study in Rabbit Eyes
}

\author{
Vassilios Kozobolis ${ }^{1}$, Maria Gkika ${ }^{1}$, Haris Sideroudi, ${ }^{1, *}$ Efthymia Tsaragli', Stylliani Lydataki ${ }^{2,},{ }^{3}$, \\ Irini Naoumidi ${ }^{3}$, Alexandra Giatromanolaki ${ }^{4}$, Dimitrios Mikropoulos ${ }^{5}$, Miguel Teus ${ }^{6}$, Georgios Labiris ${ }^{1}$
}

\author{
${ }^{1}$ Eye Institute of Thrace, Democritus University of Thrace, Alexandroupolis, Greece \\ ${ }^{2}$ Institut Carnot de Bourgogne, University of Burgundy, Bourgogne, France \\ ${ }^{3}$ Institute of Vision and Optics, University of Crete, Heraklion, Greece \\ ${ }^{4}$ Department of Pathology, Democritus University of Thrace, Alexandroupolis, Greece \\ ${ }^{5}$ 1st University Department of Ophthalmology, Aristotle University of Thessaloniki, Thessaloniki, Greece \\ ${ }^{6}$ Universidad de Alcalá, Madrid, Spain \\ * Corresponding author: Eye Institute of Thrace, Democritus University of Thrace, Alexandroupolis, Greece PC: 68100; \\ e-mail: harissid@alex.duth.gr
}

Summary: The Purpose of present study was to investigate the effect of riboflavin/ultraviolet-A-induced collagen cross-linking (CXL) on central cornea, limbus and intraocular pressure (IOP). This was an animal experimental study. The right corneas of 10 rabbits were ultraviolet-A irradiated $\left(3 \mathrm{~mW} / \mathrm{cm}^{2}\right.$ for 30 minutes $)$ after de-epithelialization and instillation of $0.1 \%$ riboflavin / $20 \%$ Dextran drops. Left corneas served as controls. Samples were examined histologically one month postoperatively. Before and after treatment, IOP measurements were recorded bilaterally. At central cornea of eyes underwent CXL keratocyte repopulation, normal arrangement of collagen fibres and a statistically significant change in fibres diameter were detected, compared to controls. At limbus area, there were not any significant histological differences after CXL. There was no statistically significant difference between pre- and postoperative IOP in all eyes.

Results indicate no pathological effects on central cornea and no significant changes in limbus and IOP after CXL in rabbit eyes, so this method might be a safe therapeutical option. Further clinical investigations may be needed.

Keywords: Corneal Crosslinking; Intraocular pressure; Limbus; Cornea

\section{Introduction}

It is known that the biomechanical properties of the cornea depend on the characteristics of the collagen fibers (1-3). The surgical technique of corneal collagen cross-linking (CXL), using ultraviolet-A (UVA) irradiation and riboflavin, affects the hydration of the cornea, the apoptosis of the keratocytes and the diameter of the collagen fibers. The CXL-associated biomechanical and enzymatic changes make the cornea stiffer and more compact after treatment (4-8). It should, also, be noted that both the instillation of riboflavin and the irradiation are applied up to the area of limbus. Among the possible additional local impact of CXL treatment are the changes in the structure of the trabecular meshwork and/or of the Schlemm's canal. The latter could be associated with a reduction of the aqueous humor outflow facility by acting in a way similar to Argon LASER Trabeculoplasty (ALT).

Based on the aforementioned hypothesis and evidence, we report our experience with experiments in rabbit eyes. Primary objective of the study was to assess potential mor- phological changes in the limbal area induced by CXL, which could affect the aqueous humor outflow and the IOP values. To our knowledge, no published data are available, regarding the impact of CXL on the limbus area and the trabeculum.

\section{Materials and Methods}

This experimental study was conducted at the Laboratory of Experimental Surgery and Surgical Research of Democritus University of Thrace, Greece, following the institutional and national guide for the care and use of laboratory animals (Trial number: EL71 BIO1), as well as principles of animal maintenance described by the Association for Research in Vision and Ophthalmology.

Ten healthy male New Zealand white albino rabbits [mean age of 5 months and mean weight of 3.6 \pm 0.3 (Mean $\pm \mathrm{SD}) \mathrm{Kg}$ ] were used for this single-center and single-surgeon experimental study. The corneal epithelium of the right eyes was mechanically removed and a $0.1 \%$ riboflavin in $20 \%$ Dextran 500 solution was instilled to the cornea for 
30 minutes, until the stroma was completely penetrated and aqueous was stained yellow. Then, the corneas were UVA-irradiated for 30 minutes (wavelength of $365 \mathrm{~nm}$, irradiance of $\left.3 \mathrm{~mW} / \mathrm{cm}^{2}\right)\left(\mathrm{UV}-\mathrm{X}^{\mathrm{TM}}\right.$ Illumination System Version 1000, IROC AG, Zurich, Switzerland). Instillation of riboflavin drops was continued during irradiation, in order to sustain the necessary concentration of the riboflavin. Moreover, balanced salt solution (BSS) was applied every 6 minutes to moisten the cornea. The left fellow eyes served as intra-individual controls, remaining completely untreated (4 eyes), with epithelial debridement alone ( 3 eyes) or with epithelial debridement plus the application of the $0.1 \%$ riboflavin / $20 \%$ Dextran solution ( 3 eyes). This was performed to exclude an influence of the epithelial abrasion or associated hydration changes in the examined tissue and especially in the trabeculum. Before all of the procedures, the rabbits were anaesthetized with subcutaneous injection of a mixture of ketamine hydrochloride $(50 \mathrm{mg} / \mathrm{Kg})$ and xylazine hydrochloride $(5 \mathrm{mg} / \mathrm{Kg})$. After CXL treatment, drops of ofloxacin, fluorometholone and diclofenac nitrate were applied on both eyes of the rabbits, until complete re-epithelization of the cornea was detected by slit-lamp biomicroscopy. Before and one month after treatment, in all animals, IOP measurements were recorded bilaterally, using the Tonopen-XL (Reichert

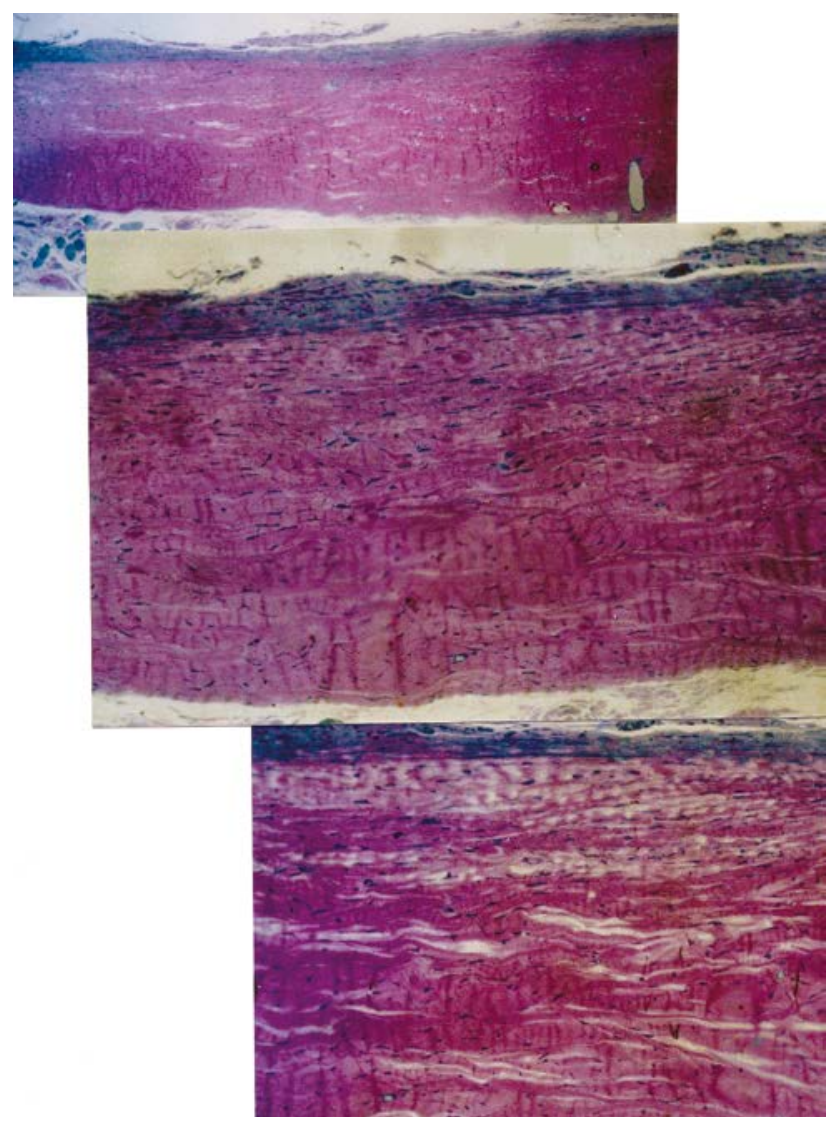

Fig. 1: Light microscope - Untreated cornea (control).
Inc., NY, USA), after topical anaesthesia (tetracaine drops), since pharmaceutical substances that are commonly used in general anaesthesia cause immediate and significant reduction of IOP (9).

The animals were sacrificed one month after completion of the experiments and both eyes were enucleated. After fixation, the corneas were excised along with a $2 \mathrm{~mm}$ scleral rim, in order to maintain normal hydration conditions and to minimise oedema and striae, and bisected. The first section of every bisected cornea was prepared for scanning electron microscopy (SEM) and the second for histologic examination (light microscopy) by trichrom staining (CytoLogix Masson Trichrome Stain Kit, Cambridge, MA). The study was observer masked.

\section{Results}

Light microscopy (Figures 1-3) showed that there was complete re-epithelialization of all corneas that underwent epithelial debridement. The CXL effect on treated corneas was established by the detection of the changes in both number and size of gaps between collagen bundles, particularly in their central area, where the treatment was focused. Limbus of the CXL treated corneas seemed to have nor-

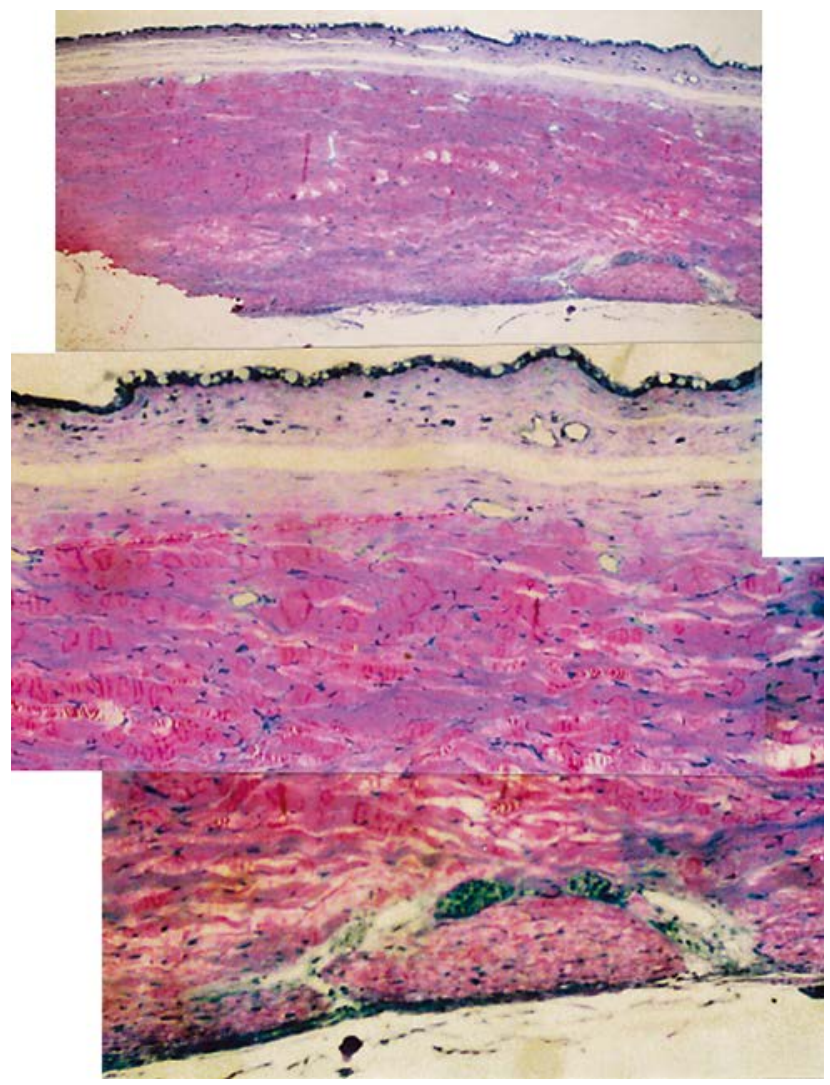

Fig. 2: Light microscope - Cornea underwent de-epithelialization and riboflavin solution instillation (control). 

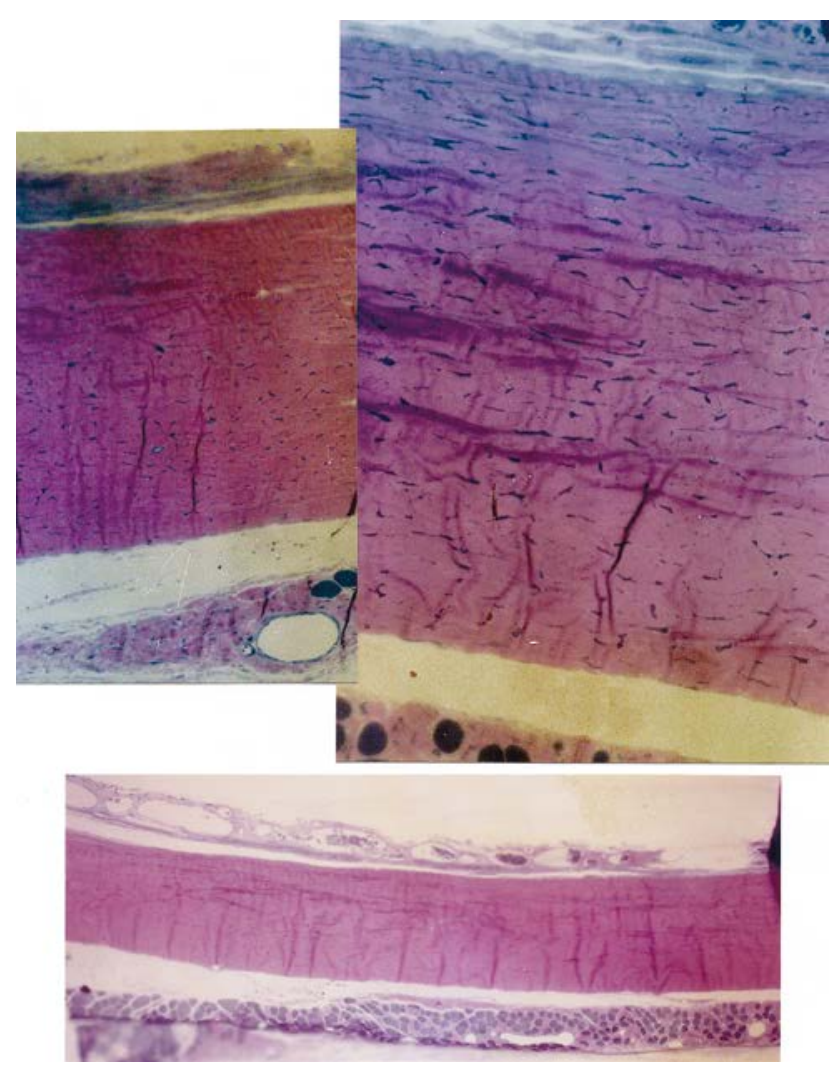

Fig. 3: Light microscope - Cornea underwent CXL.

mal appearance. Furthermore, no observable pathological changes, presence of inflammation or angiogenesis could be detected on the periphery of the corneas, including controls.

Scanning electron microscopy (Figures 4-9) showed that in all specimens that underwent debridement, a complete recovery of the corneal epithelium with a relative degree of diversity was observed. Also, in places, there was a rupture of intercellular bridges to some extent. All corneas that served as controls demonstrated a normal collagen fiber pattern with an unremarkable keratocyte appearance. On the other hand, the corneas that were treated with the CXL technique, demonstrated keratocyte repopulation of the anterior stroma, but with some acellular areas in between, as well as keratocyte apoptotic changes, such as the formation of apoptotic bodies, chromatin condensation and cell nuclei shrinkage, particularly in the periphery of the irradiated area. The structure and the arrangement of the collagen fibers seemed to be normal, with an exception of some areas of the anterior stroma, where the collagen fibers were not very well-organized in bundles. On the contrary, deeper in the stroma of the CXL treated corneas, the collagen fiber pattern was similar to that observed in controls. The distances between the collagen fibers could not be precisely evaluated due to the dehydration of the tissue before observation. However, even after dehydration, in the anterior area of central corneas, where the application of CXL treatment was focused, the changes in spaces between fibers and bundles due to therapy were evident to some extent, with these distances to seem relatively shorter compared to the rest of the cornea. In addition, there were not any significant differences at the limbus area between CXL treated eyes and controls, regarding both collagen fibers morphology and distances between collagen bundles. Finally, endothelial cells and their intracellular connections had a normal appearance in all tissue samples.

The electron microscope digital images from central cornea and limbus area of all samples were transferred to a computer screen. The collagen fiber diameters were marked manually and calculated with the help of a special semiautomatic software program (Image tool). In each image, the

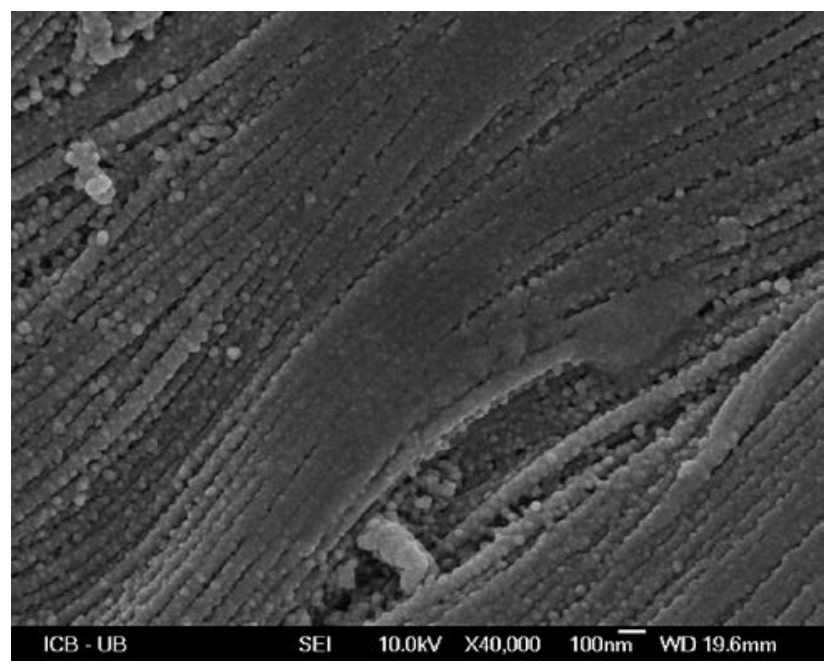

Fig. 4: SEM - Untreated cornea (control): Collagen fibers are organized in bundles. Some collagen fibers are collapsed due to fixation (arrows).

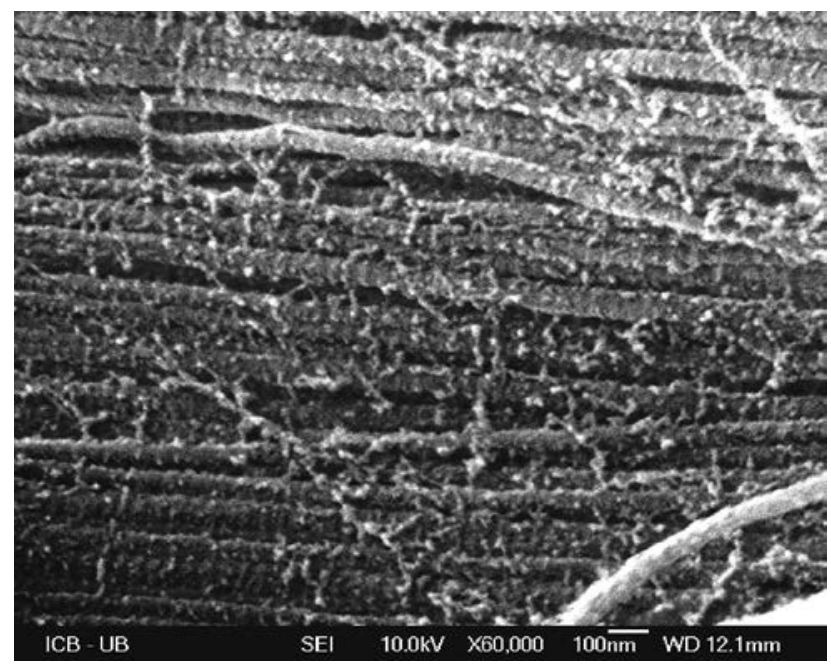

Fig. 5: SEM - Cornea underwent de-epithelialization (control): Collagen fibers with small fibrils of proteoglycans. 


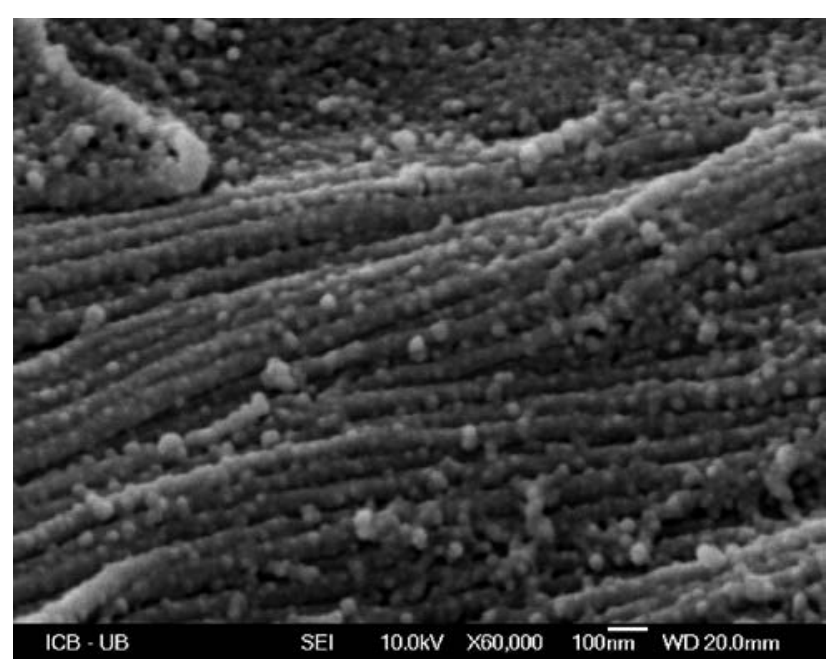

Fig. 6: SEM - Cornea underwent de-epithelialization and riboflavin solution instillation (control): Collagen fibers and granular substance (arrow).

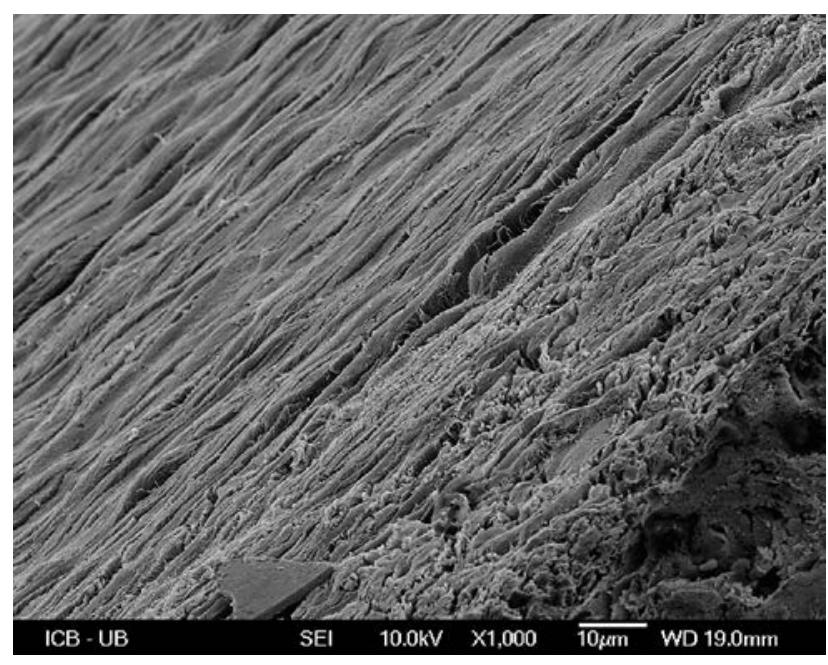

Fig. 7: SEM-Cornea underwent CXL: Sample from anterior stroma. Right arrow shows the epithelium and left arrow shows the stroma.

diameters of 20 contiguous fiber section profiles were measured. The change in thickness and morphology of collagen fibers was estimated, comparing the corneas underwent CXL treatment with controls (Table 1). Mean $( \pm \mathrm{SD}$ ) preoperative and postoperative IOP measurements of all eyes included in this study are shown in Table 2. Statistically significant difference at 0.05 level was detected using the independent samples t-test and the Wilcoxon Signed Rank Test.

\section{Discussion}

It is known that the structure of the rabbit corneal stroma is similar to human one, therefore, it was considered as the

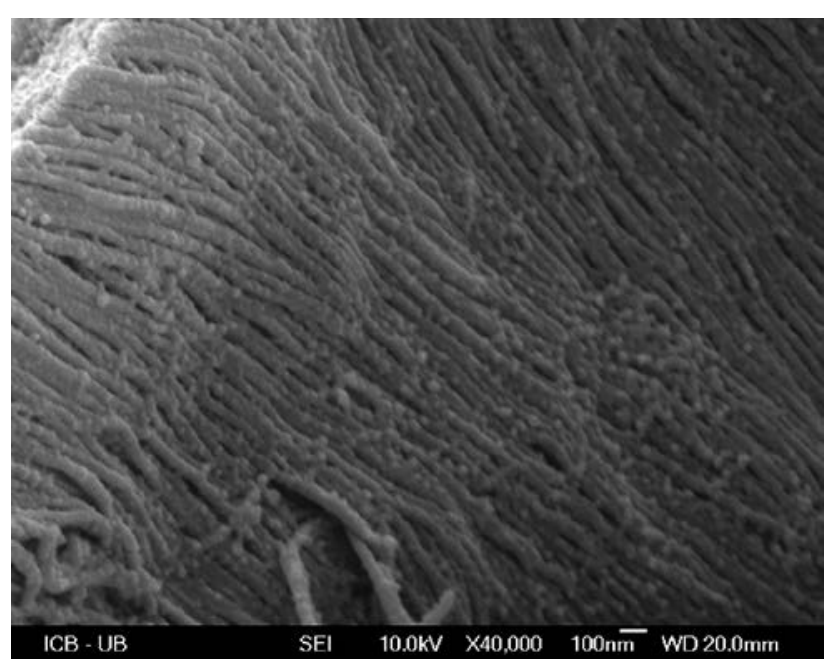

Fig. 8: SEM - Cornea underwent CXL: Sample from anterior stroma close to limbus. At some points collagen fibers are not yet well-organized in bundles and there are rests of granular extracellular substance (arrow).

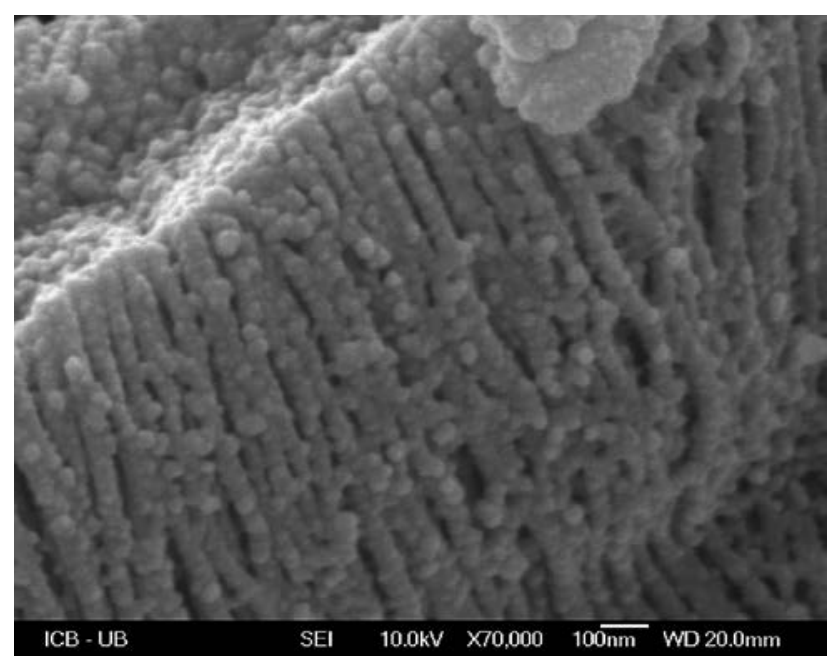

Fig. 9: SEM - Cornea underwent CXL: Sample from limbus, horizontal and perpendicular section over collagen fibers (arrows).

ideal substitute tissue for this experimental study. However, there are several differences between human and rabbit eye that have to be mentioned. The collagen fibers, which are observed in the rabbit cornea, are thinner than those in the human one. Also, other types of collagen fibres, than Type I, are found in greater frequency than in the human cornea, such as the Type VI, which usually occurs in scarring (10).

The results of this study confirmed a smooth reconstructive process following CXL treatment. Active inflammation was not observed. The epithelium regenerated rapidly after treatment and its mild non-specific differentiations seem to have no clinical significance. Regarding keratocytes, their number was normal, although some of them, especially 
Tab. 1: Preoperative and postoperative collagen fiber diameter measurements (nm).

\begin{tabular}{|l|c|c|c|c|c|c|c|}
\hline & $\begin{array}{c}\text { Without any } \\
\text { treatment }\end{array}$ & \multicolumn{2}{|c|}{ De-epithelialization } & \multicolumn{2}{|c|}{$\begin{array}{c}\text { De-epithelialization } \\
\text { + Riboflavin }\end{array}$} & \multicolumn{2}{c|}{ CXL } \\
\cline { 2 - 9 } & $($ Mean \pm SD) & $($ Mean \pm SD) & $p$ & (Mean \pm SD) & $\mathrm{p}$ & $($ Mean \pm SD) & p \\
\hline Central Cornea & $24.7 \pm 2.5$ & $26.0 \pm 2.4$ & 0.110 & $25.5 \pm 3.1$ & 0.421 & $31.4 \pm 3.5$ & $<0.001 *$ \\
\hline Limbus & $53.5 \pm 2.9$ & $53.2 \pm 2.7$ & 0.715 & $53.3 \pm 2.9$ & 0.717 & $52.3 \pm 3.9$ & 0.268 \\
\hline
\end{tabular}

*Significant at 0.05 level

Tab. 2: Preoperative and postoperative IOP measurements (mmHg).

\begin{tabular}{|l|c|c|c|}
\hline Group & $\begin{array}{c}\text { IOP pre- } \\
\text { operatively }\end{array}$ & $\begin{array}{c}\text { IOP post- } \\
\text { operatively }\end{array}$ & p \\
\cline { 2 - 3 } (Mean $\pm \mathrm{SD})$ & (Mean \pm SD) & \\
\hline CXL & $18.5 \pm 3.3$ & $20.3 \pm 3.8$ & 0.416 \\
\hline $\begin{array}{l}\text { De-epithelialization } \\
\text { + Riboflavin }\end{array}$ & $22.5 \pm 0.7$ & $19.6 \pm 0.6$ & 0.180 \\
\hline De-epithelialization & $18.6 \pm 0.3$ & $22.8 \pm 0.3$ & 0.737 \\
\hline Without any treatment & $17.4 \pm 5.1$ & $20.2 \pm 1.4$ & 0.655 \\
\hline
\end{tabular}

those located in the anterior stroma, demonstrated apoptotic features. In previous experimental studies, keratocyte apoptosis was found due to the de-epithelialization or the UVA (11-13). The clinical significance of keratocytes apoptosis is unclear. Maybe, it is associated with scarring, clouding or thinning of the cornea (14-16). This loss can be restored by repopulation due keratocyte migration from the adjacent tissue $(11,17)$. However, in our study, we observed no change in the transparency or thickness of the cornea after CXL. Regarding collagen fibers, there were no significant changes of collagen pattern and collagen fibers morphology, with an exception of some areas of the anterior stroma, in which the collagen fibers were not very well organized. This may be due to the fact that the sacrifice of the animals took place in a relatively short time after treatment. Our results showed that there is a statistically significant increase in the diameter of collagen fibers of the central cornea, indicating the effect of the CXL treatment on this area, but there was not any similar observation at the limbus area. Also, a previous study by Wollensak et al has shown a statistically significant increase in corneal collagen fiber diameter, as a result of riboflavin/UVA-induced collagen cross-linking (6). The reason for this is that the induced cross-links push the collagen molecules apart, resulting in an increased intermolecular spacing and diameter of the collagen fibers (18). However, over the years, the intrastromal processes caused a regression of the fiber thickness to baseline and a normalization of the cornea. In addition, the influence of fixation on corneal collagen has been examined systematically by others. They found an increase of the collagen fiber diameter through cross-linking induced by the fixative glutaraldehyde and a reduction in fiber diameter by the embedding resin. The two opposite effects cancel each other out, so collagen pattern is not affected by corneal fixation (19). In our series, all of the specimens underwent the same fixation, so that the relative differences between the specimens cannot have been influenced by tissue processing anyway. Also, in previous studies, there was a cytotoxic effect of CXL treatment on the endothelium, when the radiation that reached there was greater than $0.36 \mathrm{~mW} / \mathrm{cm}^{2}$. Using intensity of UVA on the surface of the cornea of $3 \mathrm{~mW} / \mathrm{cm}^{2}$, this happens, only when the thickness of the cornea is less than $400 \mu \mathrm{m}(1,20)$. In this study the endothelium remained intact, since the corneas remained transparent throughout the whole postoperative period.

Regarding limbal area our results suggested that there are not any significant differences in the distribution and morphology of collagen fibers in this area between treated and untreated corneas. There is, certainly, the case that with this cutting method and observation using SEM, we have more reliable images from the limbus rather than from the trabecular meshwork. However, we can make the assumption that, if there are no changes at the limbus area, the changes at the trabeculum are rather improbable, since the trabeculum is deeper. The rabbit cornea thickness averages $0.37 \mathrm{~mm}$ at the center and $0.45 \mathrm{~mm}$ near the limbus. Also, the cross-linking effect is not distributed homogenously over the corneal depth. The maximum effect is detected in the anterior $200-300 \mathrm{~mm}$ of the cornea, because of the UVA-absorption by the photosensitizer riboflavin $(7,21)$. This depth-dependent strengthening effect correlates with riboflavin corneal diffusion.

IOP values were measured using Tonopen-XL, since it is the tonometer of choice for measuring IOP in rabbits within the range of IOP 3 to $30 \mathrm{mmHg}$ (22). There was not any statistically significant difference between preoperative and postoperative IOP measurements. The IOP results after CXL plead for the assumption that there are no significant changes of the trabecular meshwork. This result, also, leads to the conclusion that CXL treatment might be a safe therapeutical option for patients, without influencing IOP and nerve condition.

\section{Conclusion}

In conclusion, we can say that the cornea responds well to the application of the CXL and no damage of its structure and functionality is observed. However, further experimen- 
tal and clinical studies with longer follow-up are necessary to indicate, if there are or not any significant changes at limbus area and IOP measurements after CXL, by changing some of the parameters of treatment. This information will likely be a useful tool in the design and duration of treatment and is one of our future targets.

\section{Acknowledgements/Disclosure}

No financial support was received for this study.

The authors have no actual or potential conflict of interest, including any financial, personal or other relationships with other people or organizations within three years of beginning the submitted work that could inappropriately influence, or be perceived to influence, this work.

\section{References}

1. Wollensak G, Sporl E, Seiler T. Treatment of keratoconus by collagen crosslinking Ophthalmologe 2003; 100: 44-49.

2. Wollensak G. Crosslinking treatment of progressive keratoconus: new hope. Curr Opin Ophthalmol 2006; 17: 356-360.

3. Andreassen TT, Simonsen AH, Oxlund H. Biomechanical properties of keratoconus and normal corneas. Exp Eye Res 1980; 31: 435-441.

4. Wollensak G, Aurich H, Pham DT, Wirbelauer C. Hydration behavior of porcine cornea crosslinked with riboflavin and ultraviolet A. J Cataract Refract Surg 2007; 33: 516-521.

5. Wollensak G, Spoerl E, Wilsch M, Seiler T. Keratocyte apoptosis after corneal collagen cross-linking using riboflavin/UVA treatment. Cornea 2004; 23: 43-49.

6. Wollensak G, Wilsch M, Spoerl E, Seiler T. Collagen fiber diameter in the rabbit cornea after collagen crosslinking by riboflavin/UVA. Cornea 2004; 23 : 503-507.
7. Kohlhaas M, Spoerl E, Schilde T, Unger G, Wittig C, Pillunat LE. Biomechanical evidence of the distribution of cross-links in corneas treated with riboflavin and ultraviolet A light. J Cataract Refract Surg 2006; 32: 279-283.

8. Spoerl E, Wollensak G, Seiler T. Increased resistance of crosslinked cornea against enzymatic digestion. Curr Eye Res 2004; 29: 35-40.

9. Jia L, Cepurna WO, Johnson EC, Morrison JC. Effect of general anesthetics on IOP in rats with experimental aqueous outflow obstruction. Invest Ophthalmol Vis Sci 2000; 41: 3415-3419.

10. Cintron C, Hong BS. Heterogeneity of collagens in rabbit cornea: type VI collagen. Invest Ophthalmol Vis Sci 1988; 29: 760-766.

11. Helena MC, Baerveldt F, Kim W-J, Wilson SE. Keratocyte apoptosis after corneal surgery. Invest Ophthalmol Vis Sci 1998; 39: 276-283.

12. Kim W-J, Helena MC, Mohan RR, Wilson SE. Changes in corneal morphology associated with chronic epithelial injury. Invest Ophthalmol Vis Sci 1999; 40: 35-42.

13. Pourzand C, Tyrrell RM. Apoptosis, the role of oxidative stress and the example of solar UV radiation. Photochem Photobiol 1999; 70: 380-390.

14. Nagy ZZ, Hiscott P, Seitz B, et al. Ultraviolet-B enhances corneal stromal response to 193-nm excimer laser treatment. Ophthalmology 1997; 104: 375-380.

15. Wilson SE, Kim W-J. Keratocyte apoptosis: Implications on corneal wound healing, tissue organization, and disease. Invest Ophthalmol Vis Sci 1998; 39: $220-226$.

16. Kim W-J, Rabinowitz YS, Meisler DM, Wilson SE. Keratocyte apoptosis associated with keratoconus. Invest Ophthalmol Vis Sci 1999; 69: 475-481.

17. Wilson SE. Keratocyte apoptosis in refractive surgery. CLAO J 1998; 24: 181-185.

18. Tanaka S, Eikenberry EF. Glycation induces expansion of the molecular packing of collagen. J Mol Biol 1988; 103: 495-505.

19. Fullwood NJ, Meek KM. A synchrotron X-ray study of the changes occurring in the corneal stroma during processing for electron microscopy. J Microsc 1993; 169: 53-60.

20. Wollensak G, Spoerl E, Reber F, Pillunat L, Funk R. Corneal endothelial cytotoxicity of riboflavin/UVA treatment in vitro. Ophthalmic Res 2003; 35: 324-328.

21. Spoerl E, Huhle M, Seiler T. Induction of cross-links in corneal tissue. Exp Eye Res 1998; 66: 97-103.

22. Abrams LS, Vitale S, Jampel HD. Comparison of three tonometers for measuring intraocular pressure in rabbits. Jamp Invest Ophthalmol Vis Sci 1996; 37: 940-944.

Received: 08/06/2016

Accepted: 23/08/2016 